\title{
Effi Briest: \\ Crampas und sein Lieblingsdichter Heine
}

Die seelische Unvereinbarkeit der beiden Ehepartner Melanie und Ezechiel Van der Straaten in Fontanes Roman L'Adultera wird dem Leser lange vor ihrer Scheidung durch die Verschiedenheit ihrer Kunstwelten eindringlich suggeriert. Nach Melanies Worten ist das Haus »in bilderschwärmende Montecchi und musikschwärmende Capuletti« (LA 49) geteilt. Ihr Mann ist »Bildersammler (LA 41) und schätzt vor allem die »wohltuend gemäßigte Temperatur« (LA 31) der italienischen Renaissancemalerei, während sie selbst Wagner-Musik liebt und damit - in seinen Worten - dem »Ritter von Bayreuth«, dem »Behexer Tannhäuser und Venusberg-Mann« (LA 33) zum Opfer gefallen ist, was sie von Anfang an mit ihrem späteren Liebhaber Ebenezer Rubehn verbindet, bei dessen Ankunft im Hause Van der Straaten denn auch anzüglich genug »Wotans Abschied« auf dem Klavier gespielt wird.

Auf indirekte Weise werden so durch ihre Kunstinteressen die unterschiedlichen erotischen Temperamente des Bankiers und seiner 25 Jahre jüngeren Frau und die sich daraus ergebenden Spannungen und Frustrationen signalisiert.

Auf ähnliche, wenngleich verstecktere Art steht auch Effi Briest zwischen den Kunstwelten ihres ebenfalls erheblich älteren Mannes und ihres Liebhabers, und auch in ihrem Fall deutet sich die seelische Abwendung von ihrem Mann in der Zuwendung zu einem ihm fremden Kunstinteresse an, das allerdings hier nicht in der betörenden Gewalt der romantischen Musik Wagners, sondern in dem verführerischen Zauber der Poesie Heines besteht. Die Rolle der bildenden Kunst auf der Gegenseite bleibt die gleiche, denn Innstetten begeistert sich wie Van der Straaten für italienische Malerei. Aber es gelingt ihm nie, Effis Teilnahme daran zu gewinnen, weil sich Kunst durch seinen Zugriff in totes Wissen, in das pedantische Registrieren von Fakten und in gelehrte Vorträge verwandelt.

Aus ihren Nachrichten an ihre Eltern von der Hochzeitsreise geht deutlich hervor, wie sie unter den vielen Museumsbesuchen in Italien leidet, so etwa, wenn sie schreibt: »das Nachschlagen und das lange Stehen vor den Bildern strengt mich an. Aber es muß ja sein.« (41) Ihr Vater, der ihr Leiden spürt, kommentiert ihre Bemerkungen im Gespräch mit seiner Frau auf gewohnt sarkastische Art: "Innstetten ist ein vorzüglicher Kerl, aber er hat so was von einem Kunstfex, und Effi, Gott, unsere arme Effi, ist ein Naturkind. Ich fürchte, daß er sie mit seinem Kunstenthusiasmus etwas quälen wird.« (37, Hervorhebungen hinzugefügt) Oder: »Und wenn ihr Mann, unser Herr 
Schwiegersohn, eine Hochzeitsreise machen und bei der Gelegenheit jede Galerie neu katalogisieren will, so kann ich ihn daran nicht hindern. Das ist eben das, was man sich verheiraten nennt. « (42) Natur und Kunst ... sie haben sich nicht, eh man es denkt, gefunden, sondern sie zeigen die Inkompatibilität der Ehepartner an, und Fontane stellt damit Stichworte zur Verfügung, die beide Personen insgesamt charakterisieren und den Untergang des Naturkindes Effi verstehen helfen.

Anders als ihr Mann weiß ihr zukünftiger Liebhaber Crampas auf höchst suggestive, eindringliche Weise von seinem Kunstinteresse zu sprechen und Effis Aufmerksamkeit zu gewinnen. Wo Innstetten den Gegenstand tötet, haucht Crampas ihm Leben ein, ja, es ist eins der auffälligsten Merkmale seines Umgangs mit der Literatur, daß sich durch ihn Heines Gedichte und Wicherts Lustspiel Ein Schritt vom Wege für sie regelrecht verwirklichen. Umgekehrt ist es ebenso bemerkenswert, daß andere Literatur, die sie nicht mit Crampas verbindet, sondern Unterstützung gegen die Verführung bedeuten könnte, stumm und wirkungslos bleibt wie Brentanos Gedicht von der »Gottesmauer « und Körners und Schillers Gedichte (s. u.). Es ist deshalb auch nicht übertrieben zu sagen, daß Crampas Effi im 17. Kapitel des Romans durch Poesie geradezu verführt. ${ }^{1}$

Innstettens Vorliebe für die Malerei steht also Crampas literarischem Interesse gegenüber, und der Leser erfährt in der Szene, die Effi im Rückblick als den Beginn ihrer Beziehung zu dem Major betrachtet - »Das war der erste Tag; da fing es an.« (218) -, aus ihrem Mund, daß er »neben vielem anderen gelegentlich auch Dichter (126) ist, ja, gerade durch diese Bemerkung wird zum ersten Mal ein Geheimnis zwischen beiden hergestellt, von dem Innstetten ausgeschlossen ist. Gieshübler nämlich hat Effi geschrieben, daß Crampas dichtet, und Innstetten, sagt sie zu dem Major, »braucht« den Brief »nicht zu lesen, der hat keinen Sinn für dergleichen« (125).

Warum die poetische Welt, die Crampas ihr eröffnet, Effi so anzieht, wird deutlicher, wenn man seine Begründung hört, weshalb ihm Heine so gut gefällt: »Bei Heine liegt es aber anders: alles ist Leben, und vor allem versteht er sich auf die Liebe, die doch die Hauptsache bleibt. Er ist auch sehr für das Romantische, was freilich gleich nach der Liebe kommt und nach Meinung einiger sogar damit zusammenfällt.« (137) In Heine also vereinigen sich 3 Elemente zu einer faszinierenden Mischung: Leben, Liebe und das Romantische. Mit ihnen aber ist genau der Bereich umschrieben, den Effi in ihrer Ehe mit Innstetten entbehrt. Die Pedanterie und Nüchternheit, mit denen er sein Kunstinteresse verfolgt, bestätigt nur die Entbehrungen ihres ehelichen Daseins. Crampas und sein Lieblingsdichter Heine dagegen bilden das psychische Äquivalent zu ihrer physischen Vorliebe für das Schaukeln: das Spielerische, die Fantasie Beschäftigende, den rosigen Schimmer (s. u.), den die Poesie der prosaischen Wirklichkeit verleiht. Frau von Briest fragt sich 
bezeichnenderweise schon am Tage nach Effis Hochzeit: »ihr Ehrgeiz wird befriedigt werden, aber ob auch ihr Hang nach Spiel und Abenteuer? Ich bezweifle.« (40)

Innstetten möchte diese Welt der verführerischen Literatur lieber von Effi fernhalten. Als sie ihn auf der Hochzeitsreise nach einem Mephisto-Vers aus Goethes Faust fragt, den er gerade zitiert hat, speist er sie kühl ab: „Schließlich aber sagte er, es sei eigentlich ganz gut und ein Vorzug, wenn ich nichts davon wüßte.« (41)

Die künstlerischen Gegenwelten der beiden Männer - die Bilderbegeisterung des »Kunstfex «Innstetten und die literarischen Bedürfnisse des "Dichters « Crampas - werden von Fontane so aufeinander bezogen, daß sie sich wechselseitig ausschließen müssen. Im Spätherbst nämlich schlägt Innstetten vor, »die Italienische Reise, an der Hand seiner Aufzeichnungen, noch einmal durch[zu]nehmen. Eine solche Rekapitulation sei eigentlich die Hauptsache« (143). Die trockene Nachbetrachtung ist keineswegs nach Effis Geschmack, denn »sie war nicht für Aufgewärmtheiten « (144). Die italienischen Abende aber werden, kaum daß sie begonnen haben, von der Literatur regelrecht verdrängt, bei der Crampas die entscheidende Rolle spielt, denn als die ersten beiden dieser abendlichen Kunstbetrachtungen vorüber sind und die dritte bevorsteht, fragt Gieshübler, ob Effi »nach einem Crampasschen Plane « in einer Liebhaberaufführung des Lustspiels Ein Schritt vom Wege »die Rolle der Ella«, die Hauptrolle also, zu spielen bereit sei. Effi war wie elektrisiert; was sollten Padua, Vicenza daneben bedeuten!« Sie sehnt sich nach einem »Wechsel der Dinge« (144), was weitergehende Bedeutung hat, denn sie wechselt damit zugleich von Innstetten zu Crampas hinüber.

Ernst Wicherts (1831-1902) 1872 uraufgeführtes und dabei von dem Theaterkritiker Fontane durchaus positiv besprochenes Lustspiel Ein Schritt vom Wege mit dem für Effis Geschichte so beziehungsreichen Titel bildet ein Beispiel dafür, wie der moderne Leser Fontanes in Gefahr ist, bestimmte Aspekte der indirekten Aussage in dessen Romanen zu übersehen, weil so viele der darin künstlerisch fungierenden literarischen Werke des 19. Jahrhunderts, die ihre Zeit nicht überlebt haben, ihm nicht mehr gegenwärtig sind. Will der heutige Leser sich aber nicht Dimensionen der gestalterischen Subtilität in Fontanes Romanen verschließen, dann kann er auch auf die Kenntnis dieser unterdessen obskuren Werke nicht verzichten, weil gerade dieser Romancier bestimmte Kunstwerke niemals zufällig verwendet, sondern ihnen immer vielfältige Spiegelungs- und Deutungsfunktionen im Symbolgefüge des Ganzen zuweist. 
Der Inhalt des Stückes kann in Fontanes eigenen Worten aus der genannten Theaterrezension wiedergegeben werden:

Erbprinz Egon, eh er die Herrschaft über das ihm zugefallene Fürstentum Sulzingen antritt, gedenkt inkognito seine Territorien zu bereisen. Er kann dies um so eher, als sich niemand in seinem Ländchen entsinnt, ihn je gesehen zu haben, ihn, den die Zerstreuungen und Abenteuer einer großen nachbarlichen Residenz bisher von Sulzingen fernhielten. Auch jetzt flüstert man sich gerüchtweise zu: er mache die Inkognitoreise mit einer Opernsängerin.

Gleichzeitig mit dem Erbprinzen, nunmehrigem Fürsten Egon, treffen übrigens ohne alle Beziehungen zu diesem letzteren, wenigstens zunächst - auch Arthur und Ella von Schmettwitz in Sulzingen, respektive in dem neu kreierten klimatischen Kurort des Fürstentums, in Bad Kieferthal ein. Ella, voll aristokratischer Abneigung gegen die große Heerstraße, zugleich voll poetisch-abenteuerlichem Verlangen nach einem >Schritt vom Wege<, sieht sich alsbald in Situationen eingeführt, die an Romantizismus nichts zu wünschen übrig lassen. Arthur, ihr Gemahl, verliert Notizbuch und Reisebarschaft, die Wirtin im »Goldenen Tannenzapfen« besteht auf Zahlung für ein verabreichtes Champagnerdejeuner, der erwartete Bruder, respektive Schwager des jungen Paares bleibt aus, mit ihm die letzten Ressourcen, so bleibt nichts anderes übrig, als Ella, die glückliche Besitzerin einer Pensions-Gnadenarie, zweier Schubertschen und eines Mendelssohnschen Liedes, zur »Signora Carlina«, einer beruihmten, aus Mailand eingetroffenen »Cantatrice«, avancieren zu lassen, um mit Hilfe eines Vokalkonzerts die Wirtshausansprüche des »Goldenen Tannenzapfens « befriedigen zu können.

Der Zufall will es, daß, während diese Dinge erst zwischen dem jungen Paare, dann unter Heranziehung Dritter und Vierter verhandelt werden, ein Zeitungsblatt, das bald von Hand zu Hand geht, die Bestätigung der bis dahin nur als Gerücht kursierenden Nachricht bringt: Fürst Egon reist inkognito mit einer Dame der großen Oper im Sulzingenschen umher. Lange Gesichter und kurze Diskussionen. Jeder Zweifel ist gelöst: Die Signora Cantatrice, die eben ihr Konzert angekündigt, bald darauf auch unter Beifall beendet hat, ist die verführerische Opernsängerin aus der Residenz und ihr Begleiter - der Fürst. Hieraus ergeben sich eine Menge ergötzlicher Szenen: Diensteifrige Huldigungen gegen Arthur von Schmettwitz als supponierten Inkognitofürsten, charaktervolle Ansprachen im Dienste guter Sitte und allgemeiner Landeswohlfahrt, moralische Verschwörungen gegen Signora Carlina, die, wie wir wissen, keine Signora, sondern Ella von Schmettwitz ist, endlich sogar Verhaftung des wirklichen Fürsten unter Verdacht des Straßenraubs, bis der schnell 
hereinbrechende Schluß alle Verwicklungen löst und die vermeintliche Cantatrice in ihre bürgerlichen und ehelichen Rechte, den regelrechten Fürsten Egon aber in die Herzen seiner treuen Sulzinger einsetzt. ${ }^{2}$

Auf verschiedene Weise beleuchtet das in Kessin aufgeführte Stück Effis Situation vor ihrem Ehebruch. Zunächst entsprechen die pedantischen Züge von Schmettwitz' denen Innstettens, während Ellas Sehnsucht »aus dem Regelrechten ins Ungewöhnliche, aus dem Verkünstelten in die Freiheit der $\mathrm{Na}$ tur [Hervorhebung hinzugefügt] aus dem Konventionellen ins Abenteuerliche« (4. Akt, 8. Szene) Effis ganz ähnliche Bedürfnisse spiegeln; und wie Ella als Signora Carlina mit der Welt der Kunst in Beziehung tritt, die sie dem Seitensprung gefährlich nahebringt, so wird Effi durch ihr Rollenspiel als Darstellerin in gerade diesem Stück mit Crampas durch die Literatur in Verbindung gebracht.

Crampas weigert sich bezeichnenderweise, die Rolle des Ehemanns bei der Aufführung zu übernehmen, für die er vorgesehen ist, und gibt damit indirekt zu erkennen, daß er sich seine Freiheit bewahren will und ihn die Liebschaft mit Effi zu nichts verpflichten soll, was später Wirklichkeit wird, denn, wie der Leser im Rückblick aus der von Innstetten entdeckten Korrespondenz zwischen seiner Frau und dem Major erfährt, hat er es abgelehnt, mit ihr zu fliehen: »Fort, so schreibst Du, Flucht. Unmöglich. Ich kann meine Frau nicht im Stich lassen, zu allem anderen auch noch in Not. Es geht nicht [...].« (233)

Bei der Aufführung hat er »nur die Regie übernommen«, dirigiert also Effi nach seinen Wünschen, und sie ahnt, was das bedeutet: »der Major hat so was Gewaltsames, er nimmt einem die Dinge gern über den Kopf fort. Und man muß dann spielen, wie er will, und nicht, wie man selber will.«(144)

Innstetten - wie könnte es anders sein - ist am Theaterspielen nicht beteiligt, sieht sich aber die Aufführung an. Er ist stolz auf seine hübsche junge Frau, die sich doch gerade durch das Theaterspielen einen Schritt weiter vom Wege und damit von ihm entfernt hat, und findet es »eigentlich kaum zu glauben«, daß »der Dichter ein Kammergerichtsrat « (154) ist - kein Wunder, denn er ist selbst Jurist.

Effi spielt also Komödie, erst auf der Bühne, und dann in ihrem eigenen Leben, und es ist deshalb auch nicht überraschend, daß im weiteren Verlauf der Handlung das Geheimhalten ihres Liebesverhältnisses vor Innstetten ausgerechnet mit dem Wort »Komödienspiel« $(169,197)$ bezeichnet wird. Die Literatur hat sich in Leben umgesetzt, aber Effi bezahlt den Schritt vom Wege teurer als die Ella im Lustspiel, die vor dem Fehltritt bewahrt bleibt. Beider Fehler ist der gleiche: Sie gewähren dem Poetischen, Romantischen Einlaß in ihre bürgerliche Existenz. Als Ella und Arthur von Schmettwitz am Schluß des Stückes in den nüchternen Alltag zurückkehren und der Ehemann von 
seinem Schwager gefragt wird: »Wo habt ihr denn gesteckt?«, antwortet er: »In tiefster Romantik.«

Frau von Briest macht ihre Tochter schon vor der Ehe auf die Gefahren $z u$ romantischer Vorstellungen aufmerksam, als sie erfährt, daß Effi sich einen "japanische[n] Bettschirm schwarz und goldene Vögel darauf, alle mit einem langen Kranichschnabel und dann auch noch eine Ampel für unser Schlafzimmer, mit rotem Schein « wünscht. Sie denkt es sich »so schön und poetisch [sic!] alles in einem roten Schimmer zu sehen«, aber ihre Mutter ist »bewegt« und warnt sie mit dem Satz: »Die Wirklichkeit ist anders, und oft ist es gut, daß es statt Licht und Schimmer ein Dunkel gibt.« (30 f.) Ob Frau von Briest Mathilde Möhrings Verhalten gebilligt hätte? Sie bekommt eine »rosafarbene Ampel an drei Ketten« fürs Schlafzimmer zur Hochzeit, aber sie »hing sie in den Hausflur ( saische Natur wirft. Daß Effis romantische Träume von der Ehe nicht in Erfüllung gehen, zeigt sich schon bei ihrer Ankunft in Kessin. Das Haus ihres Mannes ist dazu nicht in einen rosigen Schimmer, sondern ganz demonstrativ in eine Fülle von nüchternem Licht getaucht: "Sie war wie gebannt von allem, was sie sah, und dabei geblendet von der Fülle von Licht. In der vorderen FlurhäIfte brannten vier, fünf Wandleuchter, die Leuchter selbst sehr primitiv, von bloßem Weißblech, was aber den Glanz und die Helle nur noch steigerte. Zwei mit roten Schleiern bedeckte Astrallampen, Hochzeitsgeschenk von Niemeyer, standen auf einem zwischen zwei Eichenschränken angebrachten Klapptisch [...].«(50)

Der rosige Schimmer also fehlt gerade, denn die roten Schleier der einzigen nicht brennenden Lampen gehören gerade nicht zu ihnen; und die Lampen selbst, die sie zur Hochzeit statt der roten Ampel geschenkt bekommt, sind Astrallampen, helle Petroleumleuchter, die sich dadurch auszeichnen, daß sie wenig Schatten werfen. Was für ein sprechendes Bild für Effis von der Wirklichkeit widerlegte romantische Illusionen.

Die Aufführung von Ein Schritt vom Wege bestätigt nur die poetisch-erotische Einheit, die zwischen Effi und Crampas schon hergestellt ist. Als Innstetten an den seit einiger Zeit stattfindenden Ausritten des Ehepaars mit dem Major wegen einer "Wahlkampagne (129) nicht mehr teilnehmen kann, seine Frau und Crampas sie aber fortsetzen, lenkt dieser eines Tages »Mitte November « (134) das Gespräch auf seinen "Lieblingsdichter (137) Heinrich Heine, der ja auch ein Lieblingsdichter Fontanes war $^{3}$ und beginnt damit seine Verführung durch Poesie, die das 17. Kapitel des Romans ausmacht. Betrachtet man die Heine-Gedichte, die er Effi dabei vergegenwärtigt, im Licht der sich an- 
bahnenden sexuellen Beziehung zwischen ihnen, dann steht ihr Ehebruch unausgesprochenermaßen von Anfang an unter einem unheilvollen Stern und ist voller blutiger Zeichen. Der Leser aber muß den außerordentlichen Kunstverstand Fontanes bewundern, der diese dichterischen Texte so zwingend mit dem Handlungs- und Bedeutungsgefüge seines Romans verflochten hat.

Auch die psychologische Motivierung ist wie immer bei diesem Autor sorgfältig vorbereitet. Crampas hat Effi ein Charakterbild ihres Mannes aus seiner früheren Kenntnis entworfen und ihr dabei unter anderem nahegelegt, daß Innstetten den spukenden Chinesen als »eine Art Angstapparat aus Kalkül« verwendet, ein »Erziehungsmittel«, um sie »in Ordnung zu halten«, was Effis Stolz tief verletzt: »Es fehlte jede Herzensgüte darin und grenzte schon fast an Grausamkeit.« (134)

Durch diese Episode ist in Effi ein Bedürfnis geweckt, Innstettens Kontrolle nun gerade zu entgehen, sich ihre Freiheit zu beweisen, vielleicht sogar, ihn für seine Impertinenz zu strafen. Der Dialog mit Crampas bei dem jetzt folgenden Ausritt bekommt daher von ihrer Seite etwas ausgesprochen Herausforderndes und Flirtendes. »Ich glaube wirklich «, sagt sie etwa, »Major, Sie hielten es für ganz in der Ordnung, wenn ich ihnen eine Liebeserklärung machte.« (136) Sie spielt schon hier, wie er will, und der Ausdruck »in der Ordnung «, der gerade vorher im Zusammenhang mit ihrer Reaktion auf Innstettens Erziehungskünste gefallen ist, macht deutlich, wie sie beginnt, das Verbotene als das Gebotene zu empfinden.

Bei diesem Gespräch voller Anzüglichkeiten gibt Effi das Stichwort, das zur Unterhaltung über Heine hinüberleitet, und schon dieses Stichwort selbst gehört in einen motivischen Zusammenhang des Buches und trägt zur Deutung von Effis Schicksal bei. Sie deutet auf das Meer und bemerkt: »Da liegt Vineta, da muß es liegen, das sind die Turmspitzen.« Crampas bezieht diese Bemerkung auf Heines Gedicht »Seegespenst « aus den Nordseebildern, das Effi aber gar nicht kennt. »Ich kenne überhaupt wenig. Leider.« (137)

In »Seegespenst« wird geschildert, wie in einer versunkenen Stadt »ein Mädchen«, ein »armes, vergessenes Kind « in einem »alte[n], hochgegiebelte[n] Haus / Das melancholisch menschenleer ist«, einsam sitzt. Während um sie herum »sittsam « das Leben der Stadt auf dem Meeresboden weitergeht, lebt sie »fremd unter fremden Leuten « und verbirgt sich vor dem Sprecher des Gedichts, einem Schiffsarzt, der sich aus Sehnsucht und Liebe nach ihr ins Wasser stürzen will, aber vom Kapitän des Schiffes zurückgehalten wird. Die letzte Zeile des Textes »Doktor, sind Sie des Teufels? « sollte nach einem früheren Entwurf des Romans Effi bei seinem ersten Annäherungsversuch zu Crampas sagen, »übermütig, aber ihre Stimme zitterte« (318). Wie genau in dem Gedicht Effis Lage in Kessin wiedergegeben ist, bedarf keiner weiteren Erläuterung. Crampas' Schilderung, von Effi mit einem arglosen »Das ist ja allerliebst« begleitet, entspricht aber auffälligerweise dem eben 
wiedergegebenen Inhalt des ganzen Gedichts nur zum Teil. Seiner Darstellung nach faßt den Schiffsarzt "eine Sehnsucht, auch mit in die Kirche zu gehen«, wohin sich »trippelnde Frauen in Kapotthüten « in der Wasserstadt begeben. Die zentrale Gestalt, das einsame Mädchen, spart er aus. Seine Behauptung, daß der Doktor »vor Verlangen aufschreit« und sich ins Wasser stürzen will, um mit zur Kirche zu gehen, »wenn auch bloß um der Kapotthüte willen«, ist eine Verharmlosung, die Effis sonst unbegreifliche Reaktion verständlich macht. Liebessehnsucht ist ganz offensichtlich das Motiv in Heines Gedicht. Crampas' Worte beziehen sich in dem poetischen Text auf eine der Personengruppen, die die Wasserstadt bevölkern. Während »bedächtige Männer « über den Marktplatz auf dem Weg »nach dem treppenhohen Rathaus « sind, "wo steinerne Kaiserbilder / Wacht halten mit Zepter und Schwert«, gehen »bejahrte Frauen« mit »Gesangbuch und Rosenkranz nach dem großen Dome«.

Die erwähnten Gegenstände belegen, wie genau die Lage des sehnsüchtigen Mädchens in dem alten Giebelhaus der Effis entspricht. Die Welt nämlich, in der sie vereinsamt sitzt, ist eine Welt von Rathaus und Kirche, von Kaiserbildern und »Glockengeläute«, von Szepter und Gesangbuch, von Schwert und Rosenkranz oder, auf eine einzige Formel gebracht: von Thron und Altar. Und damit erst zeigt sich, wie präzise das Gedicht in den Bedeutungszusammenhang der Handlung eingepaßt ist, der wie immer in Fontanes Romanen in der dargestellten Wirklichkeit verborgen liegt. Achtet man aber auf ihre Details, dann enthüllt sich das, was scheinbar nur realistische Umwelt, Genrebild, Wirklichkeitstreue und damit zufällig ist, als eine Welt voller Zeichen, die dem aufmerksamen Leser die geistigen Hintergründe, die psychologischen, sozialen, politischen und kulturanalytischen Tiefenschichten des jeweiligen Romans erschließen. Völlig zurecht sagt Walther Killy über Fontane: »Fontane ist der einzige deutsche Erzähler nach Goethe, dem es gelingt, in [den Gegenständen] zeichenhafte Kräfte zu wecken, ohne ihnen die scheinbare Zufälligkeit der natürlichen Erscheinung zu nehmen. $\ll^{5}$

Thron und Altar, die preußische Einheit von Königtum und rigorosem Protestantismus, stellt für Effi die tödliche gesellschaftlich-moralische Instanz dar, vor deren Richterstuhl sie versagt und in deren Atmosphäre sie zu leben unerträglich findet. Nicht umsonst bildet Reichskanzler Bismarck, die Inkarnation des Preußen, ihren niemals selbst auftretenden und daher für sie ungreifbaren Gegenspieler, der immer wieder ihren Mann von ihr entfernt und dadurch mitverantwortlich ist für die Einsamkeit Effis in ihrer Ehe. Sie selbst wird nie nach Varzin zum Fürsten miteingeladen, »der über uns entscheidet « (82), aber es ist bezeichnenderweise ein Besuch Innstettens bei Bismarck, während dessen zum ersten Mal ihr traumatisches Gefühl der Angst und Verlorenheit in ihrer Ehe ausbricht. Und nicht umsonst bildet es den Höhepunkt von Effis gesellschaftlicher Karriere, daß der preußische König und deutsche 
Kaiser selbst »auf dem Hofball gnädige, huldvolle Worte an die schöne, junge Frau« (222) richtet.

Thron und Altar als die feindlichen Mächte, die das Naturkind Effi zerstören, drängen sich zeichenhaft in ihrem Leben auf immer neue, manchmal ganz versteckte Weise auf. ${ }^{6}$ Wie bewußt Fontane Effis Schicksal in solche preußischen Zusammenhänge einordnet, erkennt man daran, daß er sie nach ihrer Scheidung in der Königgrätzer Straße wohnen und ihr Essen aus dem »Habsburger Hof « beziehen läßt. Sie befindet sich nun auf der Seite der besiegten Feinde Preußens, der Österreicher, die unter ihrem Habsburger Kaiser 1866 bei Königgrätz vernichtend geschlagen wurden. »Die Preußen haben viele Siegestage « (116), sagt Doktor Hannemann bei Annies Geburt.

Einen Höhepunkt dieser Atmosphäre von patriotisch-protestantischer Gesinnung, auf die Effi in Kessin immer wieder gestoßen wird, bildet der Ausflug ins Försterhaus von Uvaglia, auf dessen Rückfahrt sie im Schloon zu versinken droht, in der winterlichen Überschwemmung, die das Terrain »unterirdisch « (159) und daher unmerklich unter ihr aushöhlt - ein Element der den ganzen Roman durchziehenden Wassersymbolik, die schon mehrfach dargestellt worden ist und daher hier nicht ausgeführt zu werden braucht. ${ }^{7}$ Festgehalten werden muß allerdings im Zusammenhang mit Heines »Seegespenst«, daß Crampas Effi, durch ihr eigenes Stichwort Vineta veranlaßt und durch die Identifikation mit dem einsamen Mädchen im Gedicht, schon in einer vom Wasser verschlungenen Stadt sieht, bevor der Schloon ihr gefährlich wird und die Matrosen des »hundert Meter vor der Mole gescheitert[en]« (167) Schiffes zu ertrinken drohen, was sie seelisch so stark angreift. Da das Wasser für Effi das Element der Verlockung und des Todes darstellt, ist sie als Heinesches Mädchen in Crampas' Augen schon verloren, bevor die eigentliche Verführung stattfindet. Das Heine-Gedicht erweist sich damit auch als Glied in der Motivkette, die mit dem Versenken der Stachelbeerschalen im ersten Kapitel beginnt, wo Effi selbst, ihre eigene spätere Situation ahnungslos vorwegnehmend, anmerkt: »so vom Boot aus sollen früher auch arme unglückliche Frauen versenkt worden sein, natürlich wegen Untreue.« (14)

Effis psychologische Disposition zur Verführbarkeit zeigt sich unter anderem in der durch die Wassersymbolik hergestellten Beziehung zwischen Hohen-Cremmen und Kessin. Sie selbst fühlt sich ja auch bei der Beschreibung des Schloons an ihre Heimat erinnert: »Das ist ja wie in unsrem Luch.« (159) Im Försterhaus muß Effi wieder die engstirnigen religiösen Ermahnungen von Sidonie von Grasenapp anhören, von der sie schon nach ihrer ersten Begegnung eine "Atheistin « (66) genannt worden ist; wieder hält auch der konservative preußische Adel seine vaterländischen Reden, und zum Schluß singen alle das Preußenlied. Daß es aber neben diesen patriotisch-religiösen Exzessen gerade im Försterhaus eine unterschwellig oppositionelle Stimmung gibt, die Effis Lage entspricht, ist im Hinblick auf ihr dann unmittelbar folgendes 
Vergehen gegen die moralische Ordnung nur konsequent, aber offenbar bisher unbeachtet geblieben.

Nicht umsonst begibt sich die Gesellschaft in den Wald und kehrt gerade bei einem Förster ein, dessen Beruf der Wald ist, denn wenn für Effi das Wasser das Element der Verlockung, ihrer gesellschaftlichen Fremdheit und Unzuverlässigkeit ist, so der Wald der Ort des Dunkels und der Sünde. Als der Weg nach Überwindung des Schloon »mitten durch die dichte Waldmasse« führt, macht Crampas ihr seinen Liebesantrag: »Bis dahin waren Luft und Licht um sie her gewesen, aber jetzt war es damit vorbei, und die dunklen Kronen wölbten sich über ihr.« (161) Wie so häufig bei Fontane hebt auch hier gerade das scheinbar Arglose und Unverfängliche die eigentliche Bedeutung eines Sachverhalts hervor. Gieshübler schreibt am nächsten Tag an Effi: "Ich weiß nur, daß Sie dem Schloon glücklich entronnen sind: aber es blieb auch durch den Wald noch Fährlichkeit genug." (164) Später treffen sich Effi und Crampas in der Plantage, einem "zwischen der Stadt und den Dünen liegende[n] Wäldchen« (48) zu ihren Rendezvous.

Das Försterpaar scheint oberflächlich dem offiziellen Preußenbild von Thron und Altar zu entsprechen, denn die Bilder, die Frau Ring "aus einem reichen Danziger Kornhändlerhaus « (152) mit in die Ehe gebracht hat, zeigen fast durchweg religiöse Motive, bei denen der Bezug zur keuschen Mutter Gottes deutlich ist: die Marienburg, Memlings Altarbild »Das jüngste Gericht" aus der Danziger Marienkirche und zweimal das Kloster Oliva, und ihr Mann ist "ein stattlicher, militärisch dreinschauender Herr", der sich im ersten dänischen Krieg »ausgezeichnet « (150) hat. Aber es gibt Anzeichen, daß beide diesem scheinbar konformistischen Bild nicht ganz entsprechen, und Sidonie wittert auch dementsprechend etwas Unbotmäßiges in dem Försterhaus, ein Bedürfnis. »die gesellschaftlichen Ordnungen« (154) nicht anzuerkennen. Ring benehme sich, als ob er von dem skandinavischen »Sagenkönig dieses Namens« (153) abstamme. Er braucht also den König nicht anzuerkennen, weil er selbst einer ist. Aber darüber hinaus gibt »ein sehr nachgedunkeltes Porträt des alten Nettelbeck« an der Wand zu denken, der 1806 Kolberg gegen die Napoleonischen Truppen verteidigt hat und als Verkörperung des preußischen Patrioten gilt. Ring hat das Bild nur mit Widerstreben und gewissermaßen von Innstetten gezwungen gekauft: $» D$ as Nettelbeckbild ließ reichlich zu wünschen übrig.« (152) Frau Ring stammt aus einer freien Stadt und macht in der preußischen Ehe einen ausgesprochen geduckten Eindruck. Vor allem aber ist da die 14jährige Cora Ring, die kokette Tochter beider, die sich ausgerechnet »auf Onkel Crampas' Schoß« (154) setzt und deren sexueller Leichtsinn deutlich mit Effi in Verbindung gebracht wird. Die Parallele zu Effi ist unübersehbar, denn sie möchte wieder jünger sein: dann »setze ich mich auf Oberst Götzes Schoß und reite hopp, hopp«. (9) Sidonie stößt Effi denn auch wiederholt auf das jugendliche Abbild ihrer sündigen Gedanken 
hin. So wie Effi vor ihrer Sünde in Cora die leichtfertige Seite ihrer Natur gewissermaßen als Warnung gegenübersteht, begegnet ihr, wie die hintersinnig klanggleichen, doch bedeutungsverschiedenen Vornamen zeigen, nach ihrem Ehebruch in der Dänin Thora von Pentz eine Spiegelung ihrer ehemaligen Unschuld. Die moralische Gegensätzlichkeit beider ist offenbar in dem alttestamentarischen Bezug ihrer Vornamen verborgen: Cora scheint auf die Unbotmäßigkeit der Rotte Korah, »dieser gottlosen Menschen« (4. Mose 16.26), anzuspielen, die »einen Aufruhr wider den Herrn (macht)« (ebd., V. 11). Jehova befiehlt Mose, sich und sein Volk von ihnen zu trennen, damit nicht die ganze Gemeinde gestraft wird, »wenn ein Mann gesündigt hat (ebd. V. 22) und öffnet die Erde unter ihnen, daß sie lebendig hinunter in die Hölle fahren « (ebd. V. 30). Dagegen verbindet sich mit dem Vornamen Thora der Gedanke an das alttestamentarische Gesetz, um dessen Erhaltung willen die Rotte Korah untergehen muß. ${ }^{8}$

In diesen beiden verjüngten Spiegelungen Effis zeigt sich ein Gegensatz, der den ganzen Roman durchzieht und die Dimensionen ihres Ehebruchs erhellt. Es ist der Gegensatz zwischen dem Germanischen und dem Wendischen, und von hier aus fällt Licht auch auf Vineta, die im Meer versunkene Stadt, die eine wendische Siedlung war. Wenn Effi das versunkene Vineta sucht, begibt sie sich damit unbewußt schon aus der gesellschaftlichen Ordnung ins Abenteuer. In diese Welt gehört auch die flirtende und sexuell gefährdete Cora, denn in Uvaglia, wo die Rings wohnen, hat »der Sage nach ein Wendentempel « (149) gestanden. Auf das Germanische der Skandinavierin Thora wird später durch den »auf Hansa, Skandinavien und Fritz Reuter eingeschworenen« (9) Küster Jahnke ausdrücklich und zwar mit verklärtem Gesicht hingewiesen: "Ja, so sind sie, rein germanisch, viel deutscher als die Deutschen.«(217)

So wie hier steht in Effi Briest immer wieder Wendisch-Heidnisch-Archaisches dem Germanisch-Christlich-Moralischen gegenüber, und Effis Ehebruch wird symbolisch mit ersterem in Verbindung gebracht, so bei Frau von Padden, der einzigen Dame unter dem preußischen Adel um Kessin, die für Effis erotische Gefährdungen ein gewisses Verständnis aufbringt. Sie spricht offenbar aus eigener Erfahrung, hat ihre Versuchungen aber überwunden, denn sie "suchte das, was die Natur, besonders durch starke Backenknochenbildung, nach der wendisch-heidnischen Seite hin für sie getan hatte, durch christlich-germanische Glaubensstrenge wieder in Ausgleich zu bringen « (165). ${ }^{9}$ Sie sagt zu Effi unter anderem: "Man muß immer ringen mit dem natürlichen Menschen. Und wenn man sich dann so unter hat und beinah schreien möchte, weil's weh tut, dann jubeln die lieben Engel! [...] Das hat uns unser alter Martin Luther zur Erkenntnis gebracht, der Gottesmann. « (166) So auch bei der wendischen Opferstätte am Herthasee, ${ }^{10}$ die das Ehepaar Innstetten nur besichtigt, weil es »zu spät geworden [ist], um noch nach Oberam- 
mergau zu gehen« (208), also zu einer eminent christlichen Stätte. Effi selbst sagt noch kurz vor ihrem Tod: »Ich habe von der Zeit an einen Widerwillen gegen die Wenden.« (280)

Es darf für diesen Zusammenhang nicht vergessen werden, daß die den heutigen Leser eher seltsam anmutende Verbindung von christlich und germanisch zu den Stereotypen einer Zeit gehört, die, in nationalistischen Denkschemen befangen, in allem >Welschen $<$ etwas Feindliches sah. Die Zeitgenossen Fontanes begegneten daher der Doppelformel, die der Romancier kritisch-enthüllend benutzt, in arglos-affirmativer Verwendung in allen möglichen Varianten. ${ }^{11}$

Aber Effis Geschichte belegt gerade, daß diese beiden Gegenwelten nicht so säuberlich geschieden sind, wie es scheint, denn die archaische Welt ist nur äußerlich untergegangen. Sie lebt in der christlich-modernen Welt weiter, und nur scheinbar hat diese die Blutopfer der älteren Zeit beseitigt. In Wirklichkeit liegt nämlich auch dem Christentum ein Menschenopfer zugrunde, das in Oberammergau zu besichtigen ist: die Kreuzigung Jesu. Noch immer verlangen die Ordnungsmächte im Namen eines Gotteskults Unterwerfung und strafen die Lebensäußerungen derjenigen, die die Natur in sich sprechen lassen. Was wie diachrone historische Entwicklungsstufen aussieht, ist tatsächlich eine synchrone Schichtung, bei der die früheren Phasen untergründig immer anwesend sind. Das Elementar-Urtümliche im Menschen schwelt unter der Oberfläche und bricht etwa in der Sexualität immer wieder über die zivilisierte Welt wie die Flut über Vineta herein. Immerhin war der alte Fontane ein Zeitgenosse Freuds.

Die rigorose moralische Welt Preußens hat die Blutopfer nicht abgeschafft, sondern nur sublimiert oder zeitgenössisch ritualisiert. Sie werden weiter vollzogen. Innstetten muß Crampas umbringen, und Effi wird das Herz aus dem Leibe gerissen, auch wenn das Blut nur noch symbolisch aus der kleinen Wunde am Kopf ihrer Tochter fließt, durch die die alte Schuld ans Tageslicht kommt. In Annie hat sich das für Effi tödliche Syndrom von Thron und Altar verkörpert: ihr Lieblingsschulfach ist Religion und sie ist am Tag der Schlacht von Königgrätz geboren.

$\mathrm{Da}$ der Vergleich der sich auslebenden Sexualität mit der Überschwemmung Vinetas, daß also der Rückgriff auf dieses von Effi selbst gegebene Stichwort nicht unangebracht ist, läßt sich durch einen Blick auf einen früheren Roman Fontanes belegen, in dem das Motiv der versunkenen Stadt auf ganz ähnliche, aber deutlichere Weise ebenfalls auf den Ehebruch der Heldin bezogen wird. Franziska Franz in Graf Petöfy stammt aus »einer kleinen Hafen- oder Badestadt an der Ausmündung der Oder «, in deren Schilderung wie in die Kessins in Effi Briest Fontanes eigene Kindheitserlebnisse in Swinemünde eingeflossen sind. Der in sie verliebte, mit der Ostsee nicht vertraute alte ungarische Graf Petöfy denkt sich die »kleine Stadt auf einer Sandbank 
gelegen und immer in Gefahr, vom Meere verschlungen zu werden. [...] Ich denk' es mir wie Vineta, poetisch, gruselig und ewig gefährdet.« (GP 64 f.) Durch dieses Stichwort veranlaßt - »Wo bleibt Vineta? Wo bleibt der Schrekken der Elemente? « (GP 67) - schildert ihm Franziska nun ausführlich, wie die Seestadt bedroht war, wenn während ihrer Kindheit »um die Novemberzeit« - auch Effi (s. o.) sucht Vineta »Mitte November« - der »gefürchtete Nordwester « »das Flutwasser mit solcher Gewalt von draußen her in den Strom hinein(trieb)« (GP 68), daß die Stadt in Gefahr war, wie Vineta vom Meer verschlungen zu werden. Schon hier spricht aus der Angst des Mädchens vor der Flut das Unbehagen über die erwachende Sexualität. Die »Kindermuhme « hat ihr erzählt, der Nordwestwind »könn' uns wegschwemmen, und eines Tages werd' er's auch, denn er sei der eigentliche Herr hier, und wir lebten nur von seiner Gnade, und wenn er wolle, so wär' es mit uns vorbei. Ja, dann beteten wir, aber wir wußten nicht, was wir sagten, denn wir dachten nicht an Gott und Glauben, sondern bloß an unsere Not und Gefahr, und unsere Seele war nichts als Angst und Aufhorchen auf den Sturm.« (GP 67) Eines Tages ist die Gefahr zur Wirklichkeit geworden, denn "einmal - ich seh' es, als ob es gestern gewesen wäre - stieg das Wasser drüber hinaus, und im $\mathrm{Nu}$ war die niedriger liegende Stadt ein See von einem Punkte zum andern, und in unsern Flur hinein stürzte die Welle. Da schrien wir auf, denn nun erfüllte sich unser Schicksal, und wir mußten untergehen, wie Vineta untergegangen war.« (GP 68)

$\mathrm{Daß}$ die Flut, die hier über Franziska hereinbricht, wie bei Effi Briest die Vorwegnahme ihrer sexuellen Verfehlung, das Sich-Ausleben des Triebes bedeutet, das die gesittete Welt verpönt, ergibt sich im Laufe des Romans, denn dreimal taucht das Wasser auf immer direktere und bedrohlichere Weise später im Leben Franziskas wieder auf, die nun mit dem alten Grafen eine Ehe ohne Sexualität führt und sich von seinem Neffen Graf Egon Asberg angezogen fühlt. Auch in diesem Roman bildet wie in Effi Briest das reizvolle Spiel zwischen Literatur und Wirklichkeit ein Element der Deutung.

Zunächst erinnert sich Franziska, als der Herbst auf dem ungarischen Schloß Petöfys Regen mit sich bringt, an Adelbert von Chamissos Gedicht »Der Szekler Landtag «, in dem dieser nach vergeblichen Überlegungen, wie die Natur mit Menschenkraft zu beeinflussen sei, jeden Widerstand gegen den Dauerregen aufgibt und ihn gewähren läßt. Der tatsächliche Dauerregen um das Schloß macht Franziska ihre Einsamkeit und sexuelle Frustration immer stärker bewußt: »Alte Bilder zogen herauf und mit ihnen ein Gefühl unendlicher Sehnsucht. Wonach? Wohin? Und so haben wir denn nichts sicher als ein ewig ungestilltes Verlangen? « Eines nachts tritt sie auf den Balkon hinaus und erkennt an »ein[em] dumpfe[n] Donnern und Brausen«, daß »der unter den tagelangen Regengüssen angeschwollene Bergbach [...] über die Klippenwand in die Tiefe schoß«. Während sie der Überschwemmung zuhört, erinnert 
sie sich zugleich wieder an Literatur, an Nikolaus Lenaus Gedicht »Nach Süden «, in dem sich der Geliebte mit einem Gewitter nach Ungarn sehnt, wo die Geliebte auf ihn wartet:

Aber unausgesetzt hörte sie von der Klippenwand her das eintönig mächtige Rauschen, und dazu klang es plötzlich und erinnerungsvoll in ihrer Seele:

>Hörbar rauscht die Zeit vorüber

An des Mädchens Einsamkeit.< (GP 134 f.)

Hat hier Franziska noch ihren festen Platz hinter dem Balkongitter, von dem sie symbolisch eingesperrt wird, hört sie hier noch sehnsüchtig das Wasser an sich vorüberrauschen, so begibt. sie sich bald darauf auf das Wasser selbst und kommt damit unmittelbar in Gefahr, denn bei einer Bootsfahrt mit Graf Egon droht ihr Boot in einem »Wetterloch [...] infolge beständig kreisenden Luftströmungen« in »eine von den Schiffern gefürchtete Trichterbewegung, ein[en] Strudel « gezogen zu werden und unterzugehen. Das Vinetaschicksal bedroht sie nun unmittelbar. Mit Mühe retten sich beide auf eine Insel zum Übernachten - das ist der Beginn ihres ehebrecherischen Verhältnisses. Franziska erkennt im Rückblick: »Aber die langen Regentage sind schuld, da fing es an." (GP 164) Auch in Graf Petöfy also löst das Stichwort Vineta wie in Effi Briest eine Motivkette aus, deren Funktion es ist, die erotische Gefährdung der Heldin, ihr langsames Gleiten in den Ehebruch zu veranschaulichen und zu spiegeln, so daß die äußere Natur zum Deutungsmedium der seelischen Vorgänge wird; auch hier bildet dabei die Literatur ein Element des Zusammenhanges.

Noch einmal taucht später in dem Roman die Überschwemmung auf, aber diesmal in eigenartiger und auffälliger Veränderung. Der betrogene Petöfy, der sich zum Selbstmord entschließt, als er von dem Ehebruch seiner Frau erfährt, beobachtet ein bestürzendes Naturschauspiel:

Er ritt an Plätzen vorbei, daran sich hundert Erinnerungen für ihn knüpften, bis er zuletzt auf eine künstlich aufgeworfene Höhe gekommen war, von der aus man einen Wiesengrund übersah, eine Niederung mit Tümpeln und Wasserlachen und ein paar schmalen Sandstreifen dazwischen. Eine der Lachen hatte Zufluß aus einem Graben, und das Wasser stieg infolge davon so rasch, daß es nicht bloß die Sandstreifen, sondern zugleich auch eine hier eingenistete zahlreiche Kolonie von Feldmäusen mit Überschwemmung und Untergang bedrohte. $\mathrm{Zu}$ hundert und aber hundert kamen sie von links und rechts her aus ihren Löchern hervor, um sich auf eine höhergelegene Stelle hin zu retten. Aber kaum daß sie sich hier gesammelt hatten, so schoß auch schon von einer danebenstehenden 
und in ihrer ganzen oberen Hälfte mit Nestern überdeckten Pappel allerlei Krähenvolk auf die geflüchteten Mäuse nieder und fuhr mit ihnen als gute Beute davon.

Der alte Graf hatte sein Pferd angehalten, um dem sonderbaren Schauspiele zuzusehen. ,Überall dasselbe: keine Flucht vor dem, was einmal beschlossen.<(GP 182)

Die Flutszene hat sich zum abstoßenden Bild des Tötens in der Natur gewandelt. Seine Botschaft ist klar: Wer sich aus der Flut retten kann, ist trotzdem nicht entkommen. Die Szene bildet ein eindrucksvolles Beispiel für Fontanes Technik der sprechenden Bilder.

\section{4}

So enthüllt sich das 1 . der von Crampas zitierten Heine-Gedichte als mit den zentralen Themen des Buches aufs engste verknüpft; es bildet ein Element der Deutung des Romans. Aber die Motive sind so ineinander verzahnt, daß genau an der gerade erörterten Stelle das 2. Gedicht anschließt, denn in ihm wird der Opferkomplex erörtert. Es handelt sich um »Vitzliputzli« aus dem Romanzero, und wieder ist Crampas' Darstellung verharmlosend und läßt das Entscheidende aus, so daß der Leser zum Heine-Text selbst greifen muß.

Heines 151 Strophen langes Gedicht schildert, wie sich der Autor aus dem dekadenten Europa vom »Kirchhof der Romantik [...] von verschimmelten Symbolen und versteinerten Perücken« in die naive gesunde Welt Amerikas begibt, um den Entdeckungs- und Eroberungszug von Cortez in Mexico zu vergegenwärtigen. Aber das Paradies verwandelt sich in einen Alptraum, die Unschuld ist eine Illusion - die langschnäbligen Kraniche, die Effi sich gemalt auf dem Bettschirm zur Hochzeit wünscht (s. o.), bevölkern hier die Landschaft -, denn die Spanier betrügen in ihrer Goldgier die Mexikaner. In einer Schlacht werden »über achtzig « spanische Soldaten gefangengenommen und VitzliputzIi, "Mexikos blutdürst'ge[m] Kriegsgott«, geopfert, indem ihnen bei lebendigem Leibe das Herz herausgeschnitten wird, wie Crampas berichtet: »Das war da nicht anders, Landessitte, Kultus, und ging auch alles im Handumdrehen, Bauch auf, Herz raus.« (138) Bei Heine heißt es:

»Menschenopfer« heißt das Stück.

Uralt ist der Stoff, die Fabel;

In der christlichen Behandlung

Ist das Schauspiel nicht so gräßlich.

Denn dem Blute wurde Rotwein, 
Und dem Leichnam, welcher vorkam,

Wurde eine harmlos dünne

Mehlbreispeis' transsubstituieret -

Diesmal aber, bei den Wilden,

War der Spaß sehr roh und ernsthaft

Aufgefaßt: man speiste Fleisch,

Und das Blut war Menschenblut.

Heine stellt also eine Beziehung zwischen dem Blutopfer der Heiden und dem christlichen Abendmahl her. Prinzipiell geschieht in den ursprünglichen heidnischen Kulturen dasselbe wie in der christlichen Welt. Was das mit Effis Schicksal zu tun hat, wird in dem Schlußdialog des Opferpriesters mit seinem Gott Vitzliputzli ganz deutlich. Der mexikanische Gott sieht den Untergang seines Volkes voraus; die christliche "Himmelsfürstin «, »eine Jungfrau sonder Tadel«, wird die finsteren Gottheiten Mexikos besiegen, aber er, Vitzliputzli, wird sich rächen:

Doch ich sterbe nicht; wir Götter

Werden alt wie Papageien, Und wir mausern nur und wechseln

Auch wie diese das Gefieder.

Nach der Heimat meiner Feinde,

Die Europa ist geheißen,

Will ich flüchten, dort beginn ich

Eine neue Karriere.

Ich verteufle mich, der Gott

Wird jetzund zum Gottseibeiuns;

Als der Feinde böser Feind,

Kann ich dorten wirken, schaffen.

Quälen will ich dort die Feinde, Mit Phantomen sie erschrecken Vorgeschmack der Hölle, Schwefel Sollen sie beständig riechen.

Ihre Weisen, ihre Narren

Will ich ködern und verlocken;

Ihre Tugend will ich kitzeln,

Bis sie lacht wie eine Metze. 
Ja, ein Teufel will ich werden, Und als Kameraden grüß ich

Satanas und Belial, Astaroth und Beelzebub.

Dich zumal begrüß ich, Lilis, Sündenmutter, glatte Schlange! Lehr mich deine Grausamkeiten Und die schöne Kunst der Lüge!

Mein geliebtes Mexiko, Nimmermehr kann ich es retten, Aber rächen will ich furchtbar Mein geliebtes Mexiko.

Vitzliputzli wird zum europäischen Teufel, zum Versucher, zum Gegenspieler der über die Keuschheit wachenden Jungfrau Maria, und die Schlange der Ursünde bittet er um Rat und Belehrung. Daß Effi aber im Laufe des Romans von der unschuldigen Maria zur sündigen Eva wird und daß Vitzliputzli, der versucherische Teufel, in ihrem Leben die Gestalt Crampas' angenommen hat, braucht hier nicht ausgeführt zu werden, weil es in der Fontane-Literatur schon dargestellt worden ist. ${ }^{12}$ Wieder also deutet das von Crampas zitierte Heine-Gedicht Effis Schicksal, wieder erweist es sich auf eine hintergründige Weise mit anderen Motivketten des Buches verbunden, wieder stellt sich das symbolische Gewebe des Romans als erstaunlich dicht und schlüssig heraus.

Schon in diesem Gedicht ist die blutige Atmosphäre so überwältigend, daß Effi es »indezent und degoutant zugleich« (138) findet. Aber das hindert sie nicht daran, sich gleich darauf beim Frühstück in den Dünen das 3. Heine-Gedicht erzählen zu lassen, eine Ambivalenz, die ihre Situation und den Reiz des Verbotenen spiegelt. Sie ist während der ganzen Szene zwischen Neugier und Anstand hin- und hergerissen.

Der 3. poetische Text ist auf noch bedrohlichere Weise mit Blut und Tod und Effis Leben verknüpft, denn es handelt sich in den »Spanischen Atriden«, ebenfalls aus dem Romanzero, um eine Liebesgeschichte, bei der der Liebhaber durch den Ehemann der Frau umgebracht wird. ${ }^{13}$ Crampas charakterisiert daher auch ganz folgerichtig: »Fortsetzung oder Seitenstück zum Vitzliputzli [...] nur viel pikanter, weil Liebesgeschichte.« (139) Deutet Vitzliputzli zum Schluß auf die Versuchung hin, so handelt »Spanische Atriden« von der vollzogenen Tat und ihren Folgen. Auch daß Effi zu Beginn dieser Erzählung an die 6 Frauen Heinrichs VIII. von England denkt, weist auf prekäre eheliche Beziehungen mit schrecklichen Folgen hin. 
Die Frau des Königs Pedro von Spanien, den Crampas als »grausam« und »Neidhammel« bezeichnet und verurteilt, weil er »um ein Erkleckliches zu weit« (140) gegangen ist, den Effi aber verteidigt, liebt den »schönen Kalatravaritter« Don Fredrego. Während des Festmahls, bei dem er Ehrengast sein sollte, läßt der König ihn köpfen. Aber er wird von dem treuen Hund des Hingerichteten verklagt, denn der Neufundländer trägt das abgeschlagene Haupt in den Saal und hält es der Gesellschaft entgegen.

Crampas spielt nun ganz offen die Geschichte in Effis Leben hinüber, denn er nennt den Hund Rollo, und tatsächlich übernimmt ja Rollo später dieselbe anklägerische Funktion. Auf Roswithas Bitte, den Hund nach Hohen-Cremmen zu schicken, gelangt Innstetten zu der Einsicht: "Mein Leben ist verpfuscht.« (287) Daß Crampas einerseits die Handlung des Gedichts auf Effi überträgt, aber andererseits das schreckliche Ende des Liebhabers darin nicht $\mathrm{zu}$ bedenken scheint, zeigt einmal mehr seine Sorglosigkeit, auf die er immer wieder stolz hinweist: „Wer gerade gewachsen ist, ist für Leichtsinn. Überhaupt ohne Leichtsinn ist das ganze Leben keinen Schuß Pulver wert.« (129)

Die Spiegelung von Effis Leben und Schicksal in den Heine-Gedichten wird also zunehmend direkter. Die Literatur geht gewissermaßen ins Leben über, und folgerichtig benutzt Crampas die poetischen Worte auch zur unmittelbaren Anwendung in der Wirklichkeit. Er zitiert Heines Zeile »Deine weichen Lilienfinger - eigentlich »Deine weißen Lilienfinger - aus dem Buch der Lieder, »und er berührte ihre Hand« (137). Mit Heine hat der Major sie im übertragenen Sinne verführt. Kein Wunder, daß der Dichter, den sie dem frivolen Heine entgegensetzt, der fromme Brentano, seine Gewalt in ihrem Leben verloren hat.

Sie sind so belesen, Major, aber mit einem Gedichte - freilich keinem Heineschen, keinem >Seegespenst $<$ und keinem »Vitzliputzli« - bin ich Ihnen [...] doch voraus. Dies Gedicht heißt die »Gottesmauer « [...]. Da war irgendwo Krieg, ein Winterfeldzug, und eine alte Witwe, die sich vor dem Feinde mächtig fürchtete, betete zu Gott, er möge doch »eine Mauer um sie bauen«, um sie vor dem Landesfeinde zu schützen. Und da ließ Gott das Haus einschneien, und der Feind zog daran vorüber. (151)

Dieses Gedicht versucht Effi bei der nächtlichen Rückfahrt von der Oberförsterei als Schutz gegen Crampas' Liebesantrag zu verwenden, aber Heine hat lange über Brentano gesiegt, und die Szene selbst macht es deutlich, ohne daß Fontane es mitzuteilen braucht, denn während Effi mit Brentanaos Mütterchen, aber vergebens betet, setzt Crampas nun endgültig Heines Zeile von den Lilienfingern in die Realität um: 
Gedanken und Bilder jagten sich, und eines dieser Bilder war das Mütterchen in dem Gedichte, das die "Gottesmauer « hieß, und wie das Mütterchen, so betete auch sie jetzt, daß Gott eine Mauer um sie her bauen möge. Zwei, drei Male kam es auch über ihre Lippen, aber mit einemmal fühlte sie, das es tote Worte waren. [...]

»Effi«, klang es jetzt leis an ihr Ohr, und sie hörte, daß seine Stimme zitterte. Dann nahm er ihre Hand und löste die Finger, die sie noch immer geschlossen hielt, und überdeckte sie mit heißen Küssen. (161)

Das, was Effi beim Frühstück in den Dünen vermeiden wollte, nämlich »als Reimwort auf Ihren König von Thule herum zu laufen« (142) ist nun zur Wirklichkeit geworden. Das Reimwort ist $>$ Buhle $<.{ }^{14}$ Wieder hat sich das Literarische in die Wirklichkeit umgesetzt. Ausgegangen waren beide nur vom spielerischen Zitieren von Goethes Ballade »Der König von Thule«, weil Crampas den Becher behalten wollte, aus dem Effi getrunken hat. Und auch dieser Vorgang ist Teil einer Motivkette. Daß Gieshüblers Brief mit dem Hinweis, Crampas sei ein Dichter, das erste Geheimnis Effis mit dem Major ist, ist schon erwähnt worden. Als dieser nun den Becher behalten will, droht Effi ihm: »Ich werde Innstetten davon erzählen«, worauf er kategorisch erwidert: »Das werden Sie nicht tun.« (142) Effi »schlug [...] verwirrt und fast verlegen die Augen nieder « (142). Crampas hat sie nach der Verführung durch die Poesie endgültig zu Geheimnissen vor ihrem Mann gezwungen. Das in den Heine-Gedichten dargestellte Unheil nimmt seinen Lauf.

Noch einmal allerdings tritt nach der Scheidung die machtlose literarische Gegenstimme zu Heine in Effis Leben. Bei ihrem verzweifelten Ausbruch gegen die Gesellschaft nach dem Gespräch mit ihrer Tochter und dem dann folgenden Zusammenbruch, bei dem die »Tochter der Luft« (8), die so gerne schaukelt »und am liebsten immer in der Furcht, daß es irgendwo reißen und brechen und ich niederstürzen könnte « (34), tatsächlich »am Boden, das Gesicht abgewandt, wie leblos « (275) liegt und sich ihre Entwicklung in dieser einen physischen Geste versinnbildlicht, heißt es: »Da neben dem Fenster war ein Bücherbrett, ein paar Bände von Schiller und Körner darauf, und auf den Gedichtbüchern, die alle gleiche Höhe hatten, lag eine Bibel und ein Gesangbuch.«

Theodor Körner, der patriotische Dichter der Freiheitskriege, der sein Leben für das Vaterland opferte, der idealistische Schiller, den die 2. HäIfte des 19. Jahrhunderts zu dem vaterländischen Autor, zum Inbegriff von moralischer Haltung und heroischer Selbstüberwindung stilisierte, und die religiösen Texte formen eine dem Leser wohlbekannte Einheit. Noch einmal, nun literarisch, bilden sie im Augenblick von Effis tiefstem Fall die Einheit von Patriotismus, Moral und Religion, von Thron und Altar. Das teuflische Element, das früher von dem Versucher Crampas dargestellt wurde, sieht Effi nụn aber 
woanders. In einer exorzistischen Geste legt sie »Bibel und Gesangbuch auf den Tischrand, gerade da, wo Annie gestanden hatte (274). Ihre eigene Tochter, Inkarnation des preußischen Charakters, hat teuflische Züge für sie angenommen.

\section{Anmerkungen}

${ }^{1}$ Die Formulierung wird auf Englisch von Donald C. Riechel in seinem ausgezeichneten, genauen Aufsatz »Effi Briest and the Calendar of Fate « (The Germanic Review 68, 1973, pp. 189-211) schon verwendet: "Heine thus becomes an instrument of seduction « (p. 195), »all the seductions of Heine's poetry « (p. 197). Auf die Heine-Gedichte geht Riechel allerdings fast gar nicht ein.

${ }^{2}$ Vgl. NFA XXII/1, S. 201-203.

${ }^{3} \mathrm{Vgl}$. zu dem Thema Fontane und Heine insgesamt Hans Otto Horch, » Schlechte mit demselben Vergnügen wie das Gute<. Über Fontanes Beziehungen zu Heinrich Heine«, in: Heine Jahrbuch 1979, S. 139-176. Horch diskutiert im letzten Teil seines Aufsatzes Effi Briest. Seine Ausführungen berühren sich dort mit meinen, verfolgen aber andere Spuren und deuten die Heine-Gedichte im einzelnen nicht.

${ }^{4}$ Die Zitate der Heine-Gedichte nach der Ausgabe: Heinrich Heine, Werke und Briefe. Berlin/Weimar 1972. "Seegespenst «, das kürzeste der drei von Crampas zitierten Gedichte: Bd. 1, S. 191 -193; „Vitzliputzli«: Bd. 2, S. 57-78; Spanische Atriden: Bd. 2, S. 85-95.

${ }^{5}$ Walther Killy, »Abschied vom Jahrhundert. Fontane >Irrungen, Wirrungen««, in: W. K., Wirklichkeit und Kunstcharakter. Neun Romane des 19. Jahrhunderts. München 1963, S. 204.

${ }^{6}$ Vgl. dazu im einzelnen Riechels schon erwähnten Aufsatz, S. 197-201. Dort weitere Literatur zu diesem Aspekt.

${ }^{7}$ Vgl. dazu Peter-Klaus Schuster, Theodor Fontane: Effi Briest - Ein Leben nach christlichen Bildern. Tübingen 1978 (= Studien zur deutschen Literatur Bd. 55), S. 107: "Diese Auffassung von der Staatsreligion, die nach einem Wort von Ernst Troeltsch >mehr konservativ als christlich ist $<$, kehrt jedoch im Roman immer wieder. Etwa wenn zu Annies Taufe im Toast des alten Herrn von Borcke Bismarck als $>$ Rocher de Bronce $<$ mit dem >Felsen Petri< parallelisiert wird und ihm so die Statthalterschaft Gottes auf Erden übertragen ist (268). Entsprechend ist natürlich auch der erfolgreiche Feld- 
zug gegen das französische >Sündenbabel< gottgewollt. Denn so weiß der alte Güldenklee: >Unser Alter da oben läßt sich nicht spotten, der steht zu uns.< (223) Und dieser Verbindung von Religion und Staat, nicht zur Befestigung des Glaubens, der jedem als Privatperson freigestellt ist, sondern zur Befestigung der staatlichen Ordnung, gilt auch der Toast des alten Güldenklee am Weihnachtsfest im Ringschen Hause, am Tag von Effis Sündenfall: >Mit Gott für König und Vaterland.< (302) >Kreuz und Krone< zeigt denn auch die Fahne auf Hoppensacks Hotel (333). >Kaiser Wilhelm oder Jungfrau Maria<, denkt sich Innstetten entsprechend als passenden Schmuck für die Berliner Wohnung«.

${ }^{8}$ Ich verdanke den Hinweis auf diesen alttestamentarischen Namensbezug Hans-Jürgen Schrader.

${ }^{9}$ Auch in anderen Romanen charakterisiert Fontane Gestalten ähnlich; vgl. dazu Christian Grawe: Führer durch Fontanes Romane. Stuttgart 1996, S. 13 f. (= Reclam UB 9439).

${ }^{10}$ Mit Riechel, a. a. 0., S. 198, der das Wendische und das Germanische im Herthakult vereinigt sieht, stimme ich in dieser Hinsicht nicht überein.

${ }^{11}$ Auffällig findet sie sich zum Beispiel immer wieder in den Reden zur Schillerfeier von 1859, so etwa in der Ansprache des Predigers Sydow zur Grundsteinlegung des Berliner Schillerdenkmals: »Nicht umsonst hat die Gottheit uns, den germanischen Stamm, hineingesetzt in das Herz unseres Welttheils, den germanischen Stamm mit dem tiefen, frommen Gemüth und dem hellen Auge der Forschung, mit dem Mannesschwert des Krieges und des Gedankens." (Schiller-Denkmal. Volksausgabe. 1. Bd. Berlin 1860, S. 29). Ähnlich klingt es in der Rede des Rektors der Berliner Universität Boeckh bei der Schillerfeier in der Universitätsaula: »das innere Gefühlsleben ist der schönste Schmuck edler Deutscher Frauen, ihre ächt Germanische Mitgift der Natur, gegenüber dem fremden Tand, und das Deutsche Weib, welches von Urzeiten her in dem Germanischen Leben eine würdigere Stellung eingenommen hat, [...].« (ebd., S. 55) Auch der berühmte Mediziner Virchow erging sich in seiner Rede beim Schiller-Festmahl im Krollschen Saale in Berlin in ähnlichen Gemeinplätzen: "Es ist freilich lange Zeit her, als die germanischen Stämme zuerst in das Licht der Geschichte traten und durch ihre strenge und keusche Ehe, durch ihre Schätzung weiblicher Würde das Staunen der Römer erregten.« (ebd., S. 93).

${ }^{12}$ Vgl. dazu Schuster, a. a. 0., S. 49-109. Riechel, a. a. 0., S. 199, sieht bei seinem einzigen Hinweis auf den Inhalt der Heine-Gedichte in Vitzliputzli »Wilhelminian society's cult of honor «, was mir zu einschränkend formuliert scheint.

${ }^{13}$ Ein kurzer Hinweis auf das Gedicht in Effi Briest bei Gertrude Michielsen, Die Preparation of die Future. Techniques of Anticipation in die Novels of 
Theodor Fontane and Thomas Mann. Bern u. a. 1978, S. 95, und bei Thomas Degering, Das Verhältnis von Individuum und Gesellschaft in Fontanes »Effi Briest « und Flauberts »Madame Bovary«. Bonn 1978, S. 50 f.

${ }^{14}$ Vgl. dazu Paul Anderson, "»Meine Kinderjahre $<$ die Brücke zwischen Leben und Kunst. Eine Analyse der Fontaneschen Mehrdeutigkeit als Versteck-Sprachspiel im Sinne Wittgensteins«, in: Fontane aus heutiger Sicht. Analysen und Interpretationen seines Werks, hg. von Hugo Aust. München 1980, S. 161. Der Verf. sieht in >Buhle < das Reimwort beim Versenken der Stachelbeeren im ersten Kapitel von Effi Briest. In Andersons reizvollem Aufsatz auf S. 164-167 auch Hinweise auf "Das Seegespenst « und das Vinetamotiv im Zusammenhang mit den Kinderjahren. 